\title{
Chaotic Dynamos Generated by a Turbulent Flow of Liquid Sodium
}

\author{
F. Ravelet, ${ }^{1}$ M. Berhanu, ${ }^{2}$ R. Monchaux, ${ }^{1}$ S. Aumaître, ${ }^{1}$ A. Chiffaudel,${ }^{1}$ F. Daviaud, ${ }^{1}$ B. Dubrulle, ${ }^{1}$ M. Bourgoin, ${ }^{3}$ \\ Ph. Odier, ${ }^{3}$ N. Plihon, ${ }^{3}$ J.-F. Pinton, ${ }^{3}$ R. Volk, ${ }^{3}$ S. Fauve, ${ }^{2}$ N. Mordant, ${ }^{2}$ and F. Pétrélis ${ }^{2}$ \\ ${ }^{1}$ Service de Physique de l'Etat Condensé, Direction des Sciences de la Matière, CEA-Saclay, \\ CNRS URA 2464, 91191 Gif-sur-Yvette cedex, France \\ ${ }^{2}$ Laboratoire de Physique Statistique de l'Ecole Normale Supérieure, CNRS UMR 8550, \\ 24 Rue Lhomond, 75231 Paris Cedex 05, France \\ ${ }^{3}$ Laboratoire de Physique de l'Ecole Normale Supérieure de Lyon, CNRS UMR 5672, 46 allée d'Italie, 69364 Lyon Cedex 07, France
} (Received 1 April 2008; published 14 August 2008)

\begin{abstract}
We report the observation of several dynamical regimes of the magnetic field generated by a turbulent flow of liquid sodium (VKS experiment). Stationary dynamos, transitions to relaxation cycles or to intermittent bursts, and random field reversals occur in a fairly small range of parameters. Large scale dynamics of the magnetic field result from the interactions of a few modes. The low dimensional nature of these dynamics is not smeared out by the very strong turbulent fluctuations of the flow.
\end{abstract}

Magnetic fields of planets and stars are generated by a dynamo mechanism that converts part of the work driving the flow of an electrically conducting fluid into magnetic energy [1]. The magnetic fields of Earth and the Sun involve a spatially coherent large scale component with a dipolar structure. In addition, despite the strongly turbulent nature of the flows, their dynamics are well characterized. The Earth dipole is nearly stationary on time scales much larger than the ones related to turbulence in the liquid core, but displays random reversals. Reversals occur nearly periodically in the Sun, roughly every 11 years. Direct numerical simulations have successfully displayed reversals of the magnetic field [2], but they cannot be performed in a realistic parameter range. Although laboratory experiments cannot reach the kinetic Reynolds numbers Re of the Sun or even Earth, they provide useful information about the effect of turbulence on the dynamo process. The first experimental fluid dynamos have generated either stationary or oscillatory magnetic fields [3]. Recently, the VKS experiment [4] has shown a variety of dynamical behaviors. Depending on the amount of global rotation, both stationary and time dependent regimes, including field reversals [5], have been observed. We study here the secondary bifurcations between different dynamo regimes and we show that the dynamics can be understood as the result of a few competing modes in the vicinity of the dynamo threshold.

The VKS experimental setup is sketched in Fig. 1 and has been described in [4]. A von Kármán swirling flow is generated in an inner copper cylinder (radius $R_{c}=$ $206 \mathrm{~mm}$, length $524 \mathrm{~mm}$ ) by two counter-rotating impellers $371 \mathrm{~mm}$ apart (rotation frequencies $F_{1}$ and $F_{2}$ ). They consist of soft iron disks (radius $R=154.5 \mathrm{~mm}$ ) fitted with 8 curved blades of height $h=41.2 \mathrm{~mm}$. An annulus of inner diameter $175 \mathrm{~mm}$ and thickness $5 \mathrm{~mm}$ is attached along the inner cylinder in the midplane between the disks. The fluid is liquid sodium (density $\rho \simeq 930 \mathrm{~kg} \mathrm{~m}^{-3}$, elec- trical conductivity $\sigma \simeq 10^{7} \mathrm{ohm}^{-1} \mathrm{~m}^{-1}$, kinematic viscosity $\nu \simeq 10^{-6} \mathrm{~m}^{2} \mathrm{~s}^{-1}$ ). The flow is surrounded by sodium at rest in an outer cylinder (radius $289 \mathrm{~mm}$, length $604 \mathrm{~mm}$ ). The driving motor power is $300 \mathrm{~kW}$ and cooling by an oil circulation inside the wall of the outer copper vessel allows experimental operation at constant temperature in the range $110-160^{\circ} \mathrm{C}$. The integral Reynolds numbers, defined as $\operatorname{Re}_{i}=2 \pi R^{2} F_{i} / \nu(i=1,2)$, take values up to $5 \times$ $10^{6}$. Correspondingly, magnetic Reynolds numbers, $R_{m i}=$ $2 \pi \mu_{0} \sigma R^{2} F_{i}$, up to 52 at $120^{\circ} \mathrm{C}$ are reached $\left(\mu_{0}\right.$ is the magnetic permeability of vacuum) [6]. The magnetic field is measured with Hall probes inserted inside the fluid [labeled (1) and (2) in Fig. 1]. We define $x$ the axial coordinate directed from disk 1 to disk $2, r$ the radial coordinate and $\theta$ the azimuthal coordinate. The center of the cylinder is $r=x=0$. Unless otherwise stated, measurements are performed in the midplane flush with the inner copper cylinder $[x=0, r=206 \mathrm{~mm}$, (1) in Fig. 1].

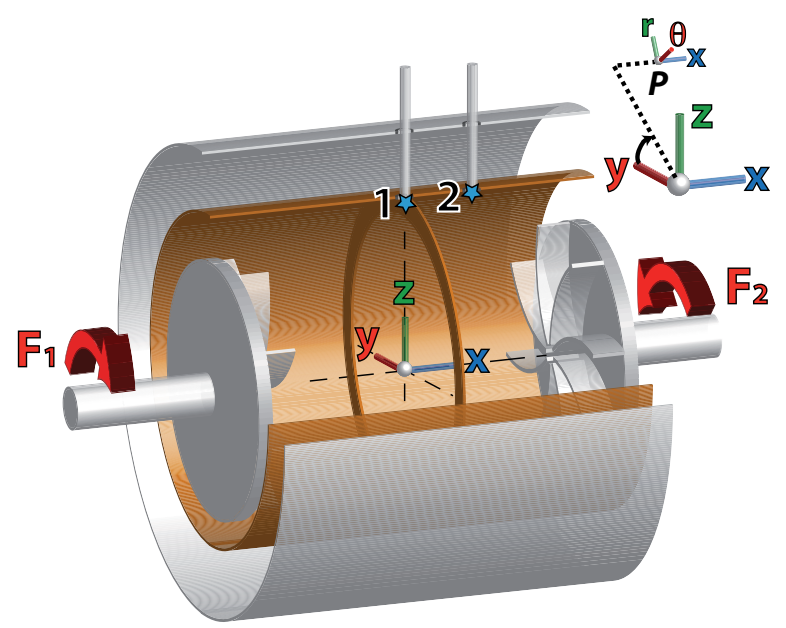

FIG. 1 (color online). Sketch of the experimental setup (see text). 
The parameter space is displayed in Fig. 2. When the impellers are counter-rotated at the same frequency, $F_{1}=$ $F_{2}=F$, a stationary imperfect supercritical bifurcation to the dynamo regime is found for a critical value $F_{c} \sim 16 \mathrm{~Hz}$ $\left(R_{m c} \sim 30\right)$ [4]. No secondary instability is observed when $F$ is increased further up to its maximum value, $26 \mathrm{~Hz}$, and the dynamics as well as the mean field geometry are unchanged. The mean field is mostly azimuthal close to the flow periphery whereas the azimuthal and axial components are of the same order of magnitude in the bulk of the flow. The radial component is much weaker. Additional measurements show that the mean magnetic field has a dominant dipolar component, aligned with the axis of rotation (see Fig. 3, top left). In contrast, various dynamical regimes are observed within a small range of $R_{m}$ (roughly $20 \%$ ), when the impellers are rotating at different speeds. This is the most striking feature of the parameter space displayed in Fig. 2. When $F_{1} \neq F_{2}$, relaxation cycles, random reversals or bursts, and oscillatory dynamos, alternate with different stationary dynamo regimes.

Starting from impellers rotating at $22 \mathrm{~Hz}$, and decreasing the frequency of an impeller, say $F_{2}, F_{1}$ being kept constant, we will describe in detail the first bifurcation from a stationary to a time dependent dynamo. As said above, we start from a statistically stationary dynamo regime with a

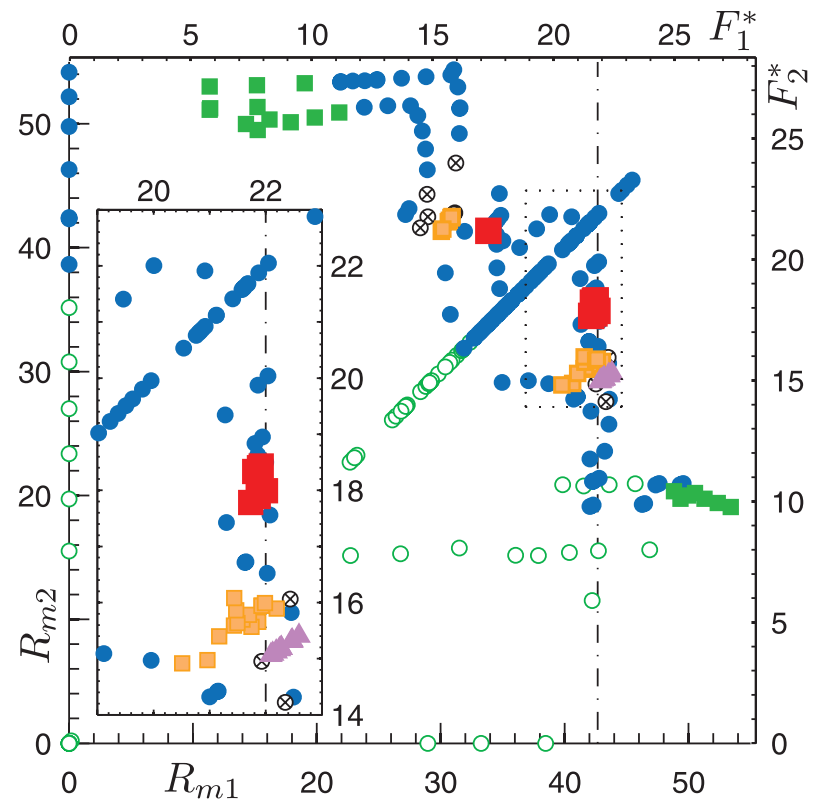

FIG. 2 (color). Observed dynamo regimes. The parameter space is labeled by the magnetic Reynolds numbers $R_{m i}$ and, for clarity, by the frequencies corrected for mean temperature variation: $F_{i}^{*}=\left[\sigma(T) / \sigma\left(120^{\circ} \mathrm{C}\right)\right] F_{i}$. Legend: no dynamo (green $\circ$; $B \lesssim 10 \mathrm{G}$ for more than $180 \mathrm{~s}$ ), statistically stationary dynamos (blue $\bullet$ ); oscillatory dynamos (green squares); limit cycles (red squares; see Fig. 3), magnetic reversals (orange squares; see Fig. 4), bursts (purple triangles; see Fig. 4) and transient magnetic extinctions $(\otimes ;$ see text). The dash-dotted line indicates the path followed here, zoomed in the inset. dominant azimuthal mean field close to the flow periphery (Fig. 3, top left). This corresponds to the trace labeled 2222 in the $\left(B_{r}, B_{\theta}\right)$ plane of Fig. 3 (middle). As the frequency of the slower impeller is decreased, we obtain other stationary dynamo regimes for which the radial component of the mean field increases and then becomes larger than the azimuthal one (22-20 and 22-19). When we tune the impeller frequencies to 22 and $18.5 \mathrm{~Hz}$, respectively, a global bifurcation to a limit cycle occurs. We observe that the trajectory of this limit cycle goes through the location of the previous fixed points related to the stationary regimes. This transition thus looks like the one of an excitable system: an elementary example of this type of bifurcation is provided by a simple pendulum submitted to
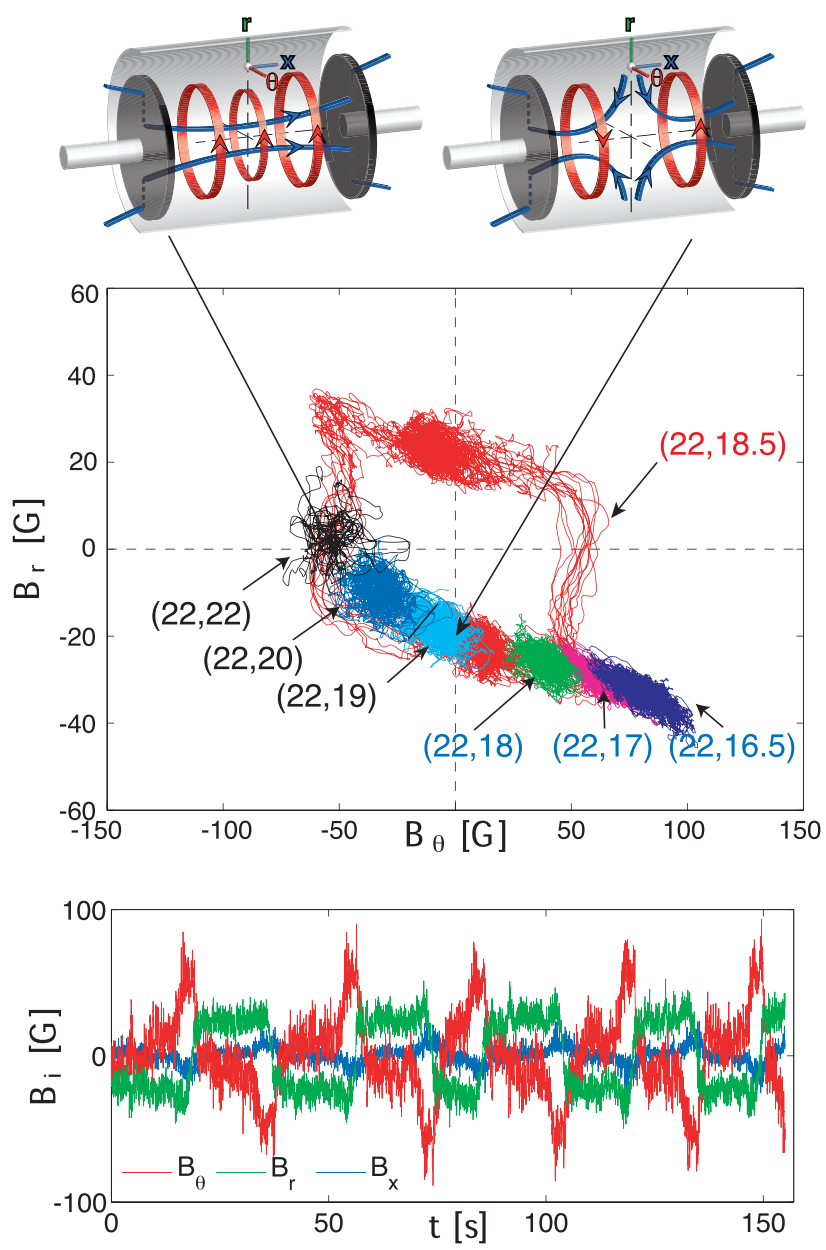

FIG. 3 (color). Top: sketch of the axial dipolar (left) and quadrupolar (right) magnetic modes. The poloidal (azimuthal) field component is displayed in blue (red). Middle: location of the different states in the $\left(B_{r}, B_{\theta}\right)$ plane: fixed points corresponding to the stationary regimes for frequencies $(F 1-F 2)$; limit cycle observed for impellers counterrotating at different frequencies $22-18.5 \mathrm{~Hz}$ (red). The magnetic field is time averaged over $1 s$ to remove high frequency fluctuations caused by the turbulent velocity fluctuations. Bottom: time recording of the components of the magnetic field for frequencies $22-18.5 \mathrm{~Hz}$. 
a constant torque. As the value of the torque is increased, the stable equilibrium of the pendulum becomes more and more tilted from the vertical and for a critical torque corresponding to the angle $\pi / 2$, the pendulum undergoes a saddle-node bifurcation to a limit cycle that goes through the previous fixed points. Direct time recordings of the magnetic field, measured at the periphery of the flow in the midplane between the two impellers, are displayed in Fig. 3 (bottom). We propose to ascribe the strong radial component (in green) that switches between $\pm 25 \mathrm{G}$ to a quadrupolar mode [see Fig. 3, top right]. Its interaction with the dipolar mode (Fig. 3, top left) that is the dominant one for exact counter-rotation, gives rise to the observed relaxation dynamics. This hypothesis is supported by measurements made outside of the equatorial plane $(x=$ $109 \mathrm{~mm}, r=206 \mathrm{~mm}$ ) where the radial to azimuthal field ratio is much smaller, as it should if the radial field mostly results from the quadrupolar component. We note that it has been often observed that dipolar and quadrupolar dynamo modes can have their respective thresholds in a narrow range of $R_{m}$ [7] and this has been used to model the dynamics of the magnetic fields of the Earth [8] or the Sun [9]. The relaxation oscillation is observed in a rather narrow range of impeller frequency $F_{2}$ (less than $1 \mathrm{~Hz}$ ). Increasing the frequency difference between the impellers, statistically stationary regimes are recovered (22-18 to 2216.5 Hz in Fig. 3, middle). They also correspond to fixed points located on the trajectory of the limit cycle, except for the case $22-16.5 \mathrm{~Hz}$ that separates from it.

When the rotation frequency of the slowest impeller is decreased further, new dynamical regimes occur. One of them consists in field reversals [5]. The three components of the magnetic field reverse at random intervals (see Fig. 4, top left, where only the azimuthal component is displayed). The average length of phases with given polarity can be 2 orders of magnitude larger than the duration of a reversal that corresponds to an ohmic diffusion time scale ( $\tau_{\sigma}=\mu_{0} \sigma L^{2} \sim 1 \mathrm{~s}$ on the scale $L$ of the experiment). We observe that the regimes with given polarity involve an amplitude of the azimuthal field in the range 50-100 G. The amplitude very slowly decays before each reversal and displays a strong overshoot immediately after. Similar features have been reported in some palaeomagnetic recordings of the Earth's magnetic field [10]. Another open question in palaeomagnetism concerns the variation of the mean frequency of reversals over the geological ages. We show here that a slight change of the fluid physical properties is enough to strongly modify this rate. Increasing the sodium temperature from 131 to $147^{\circ} \mathrm{C}$ transforms the aperiodic reversal regime of Fig. 4 (top left) into a nearly periodic one (top right) with a higher average frequency. This change in temperature decreases $R_{m}$ by less than $5 \%$ (respectively, increases Re by 7\%).

We also emphasize that, despite the strong level of turbulent fluctuations, the trajectories connecting the two
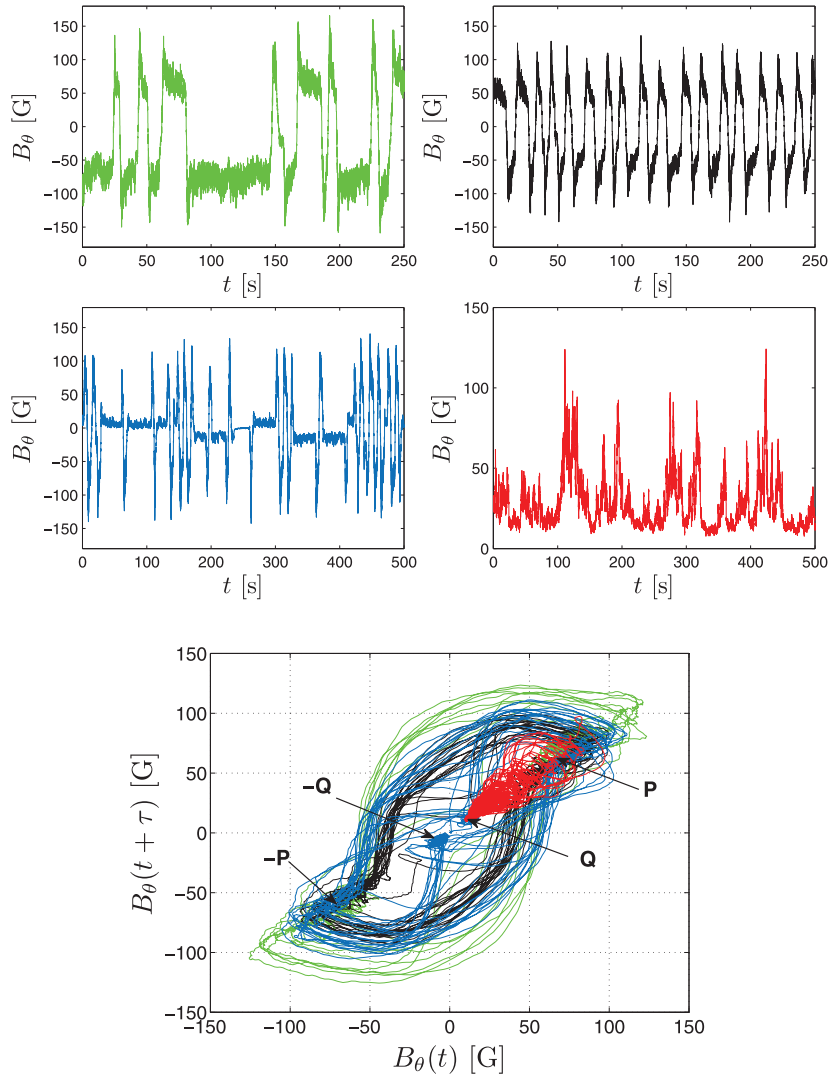

FIG. 4 (color). Top: time recordings of the azimuthal component of the magnetic field observed for impellers rotating at (2216) $\mathrm{Hz}$. The sodium temperature is $131^{\circ} \mathrm{C}$ (left) and $147^{\circ} \mathrm{C}$ (right). Middle: time recordings of the azimuthal component of the magnetic field observed for impellers rotating at $(22-15) \mathrm{Hz}$ (left), (21-15) Hz (right). Bottom: plot of a cut in phase space $\left[B_{\theta}(t), B_{\theta}(t+\delta t)\right]$ with $\delta t=1 \mathrm{~s}$ for the four regimes: in green (black) for aperiodic (nearly periodic) reversals, in blue, for symmetric bursts $(21-15 \mathrm{~Hz})$ and in red for asymmetric bursts $(22-15 \mathrm{~Hz})$. In these last two plots the magnetic field is time averaged over $0.25 \mathrm{~s}$ to remove high frequency fluctuations.

field polarities are rather robust, as shown in the twodimensional cut of the phase space displayed in Fig. 4 (bottom). For both aperiodic and nearly periodic reversals, a noisy limit cycle is obtained, the system slowing down in the vicinity of two points labeled $\pm P$ that correspond to the nearly stationary phases with a given polarity. We will show next that the fixed points $\pm P$ together with two other ones labeled $\pm Q$, are also involved in other dynamical regimes observed in the vicinity of reversals in the parameter space and shown in Fig. 4. For rotation frequencies 22$15 \mathrm{~Hz}$, the magnetic field displays intermittent bursts (middle right). The most probable value of the azimuthal field is roughly $20 \mathrm{G}$ but bursts up to more than $100 \mathrm{G}$ are observed such that the probability density function of the field has an exponential tail (not shown). For rotation frequencies $21-15 \mathrm{~Hz}$, the same type of dynamics occur, but in a symmetric fashion, both positive and negative 
values of the field being observed (middle left). Note also that the magnetic field vanished for a while $(t \sim 300 \mathrm{~s})$. Such extinctions are also observed as transient after changes of the driving in this area of the phase diagram (black circled x's in Fig. 2). In the section of the phase space displayed in Fig. 4 (bottom), we observe that the bursts occur from the unstable fixed points $\pm Q$.

The complex dynamics generated by the VKS flow can be understood as resulting from the competition between a few nearly critical modes. This has been observed in other hydrodynamical instabilities since the early experimental studies on codimension-two bifurcations [11]. Competing critical modes have been also considered as phenomenological models of the dynamics of the magnetic fields of the Earth $[8,12]$ or the Sun $[9,13]$. The same idea can be used here as follows: in the case of exact counterrotation, the axial dipole (Fig. 3, top left) can be understood as the result of an $\alpha-\omega$ mechanism [14]. The $\omega$ process results from the strong differential rotation in the case of counterrotating impellers. The $\alpha$ effect can be related to the helical nature of the flow ejected by the centrifugal force close to each impeller between adjacent blades. This non axisymmetric flow component, enhanced by the blades, is essential to bypass Cowling theorem (the mean flow alone generates an equatorial dipole as observed in [15]). It is well known in the context of mean field dynamos that dipolar and quadrupolar modes can have similar threshold values [7]. In addition, increasing the velocity difference between the impellers change the strength and/or the location of the layers with strong $\alpha$ and $\omega$ effects, and thus the threshold values of the different modes [1]. Consequently, we can understand that different dynamo modes bifurcate first for different values of $F_{1}-F_{2}$. An interesting aspect of our measurements is that we never observed a direct bifurcation from $B=0$ to an oscillatory dynamo. Time dependent regimes bifurcate from stationary dynamos, for instance via a saddle-node bifurcation (Fig. 3). This is an alternative description of oscillatory dynamos, in contrast to Parker's mechanism that involves a Hopf bifurcation. More complex time dependent regimes result from the proximity of two different stationary dynamos (Fig. 4).

The effect of competing modes on field reversals has been already emphasized in numerical simulations and mean field or analytical models [16]. It is also well-known since Rikitake that dynamo models corresponding to a drastic truncation of the governing equations can give rise to complex temporal dynamics [17]. We emphasize that what is remarkable in the present study is the robustness of these low dimensional dynamical features that are not smeared out despite large turbulent fluctuations of the flow that generates the dynamo field. A possible explanation is that the large scale magnetic field is too slow to follow velocity perturbations with time scales comparable to the rotation rate of the impellers or smaller.

We thank M. Moulin, C. Gasquet, J.-B. Luciani, A. Skiara, D. Courtiade, J.-F. Point, P. Metz, and V. Padilla for their technical assistance and the "Dynamo" GDR 2060. This work is supported by ANR No. 05-0268-03, Directions des Sciences de la Matière and de l' Energie Nucléaire of CEA, Ministère de la Recherche and CNRS. The experiment is operated at CEA/Cadarache DEN/DTN.

[1] H. K. Moffatt, Magnetic Field Generation in Electrically Conducting Fluids (Cambridge University Press, Cambridge, England, 1978).

[2] P. H. Roberts and G. A. Glatzmaier, Rev. Mod. Phys. 72, 1081 (2000) and references therein.

[3] R. Stieglitz and U. Müller, Phys. Fluids 13, 561 (2001); A. Gailitis et al., Phys. Rev. Lett. 86, 3024 (2001).

[4] R. Monchaux et al., Phys. Rev. Lett. 98, 044502 (2007).

[5] M. Berhanu et al., Europhys. Lett. 77, 59001 (2007).

[6] Note that Re and $R_{m}$ definitions are different from [4,5].

[7] P. H. Roberts, Phil. Trans. R. Soc. A 272, 663 (1972).

[8] I. Melbourne, M. R. E. Proctor, and A. M. Rucklidge, Dynamo and Dynamics, A Mathematical Challenge, edited by P. Chossat et al. (Kluwer Academic, Dordrecht, 2001), p. 363 .

[9] E. Knobloch and A. S. Landsberg, Mon. Not. R. Astron. Soc. 278, 294 (1996).

[10] J. P. Valet, L. Meynadier, and Y. Guyodo, Nature (London) 435, 802 (2005).

[11] S. Fauve et al., Phys. Rev. Lett. 55, 208 (1985); R. W. Walden et al., Phys. Rev. Lett. 55, 496 (1985); I. Rehberg et al., Phys. Rev. Lett. 55, 500 (1985).

[12] P. Chossat and D. Armbruster, Proc. R. Soc. A 459, 577 (2003).

[13] S. M. Tobias, N. O. Weiss, and V. Kirk, Mon. Not. R. Astron. Soc. 273, 1150 (1995).

[14] F. Pétrélis, N. Mordant, and S. Fauve, Geophys. Astrophys. Fluid Dyn. 101, 289 (2007).

[15] L. Marié et al., Eur. Phys. J. B 33, 469 (2003); M. Bourgoin et al., Phys. Fluids 16, 2529 (2004); F. Ravelet et al., Phys. Fluids 17, 117104 (2005).

[16] G. R. Sarson and C. A. Jones, Phys. Earth Planet. Inter. 111, 3 (1999); F. Stefani and G. Gerbeth, Phys. Rev. Lett. 94, 184506 (2005); P. Hoyng and J. J. Duistermaat, Europhys. Lett. 68, 177 (2004).

[17] T. Rikitake, Proc. Cambridge Philos. Soc. 54, 89 (1958); D. W. Allan, Proc. Cambridge Philos. Soc. 58, 671 (1962); A. E. Cook and P. H. Roberts, ibid. 68, 547 (1970); W. V. R. Malkus, EOS Trans. Am. Geophys. Union 53, 617 (1972); P. Nozières, Phys. Earth Planet. Inter. 17, 55 (1978). 